\title{
From AGB to PN in External Galaxies
}

\author{
Cesare Chiosi
}

Department of Astronomy, University of Padua, Vicolo Osservatorio 535122 Padua, Italy

\begin{abstract}
We present new results from spectro-photometric models for elliptical galaxies in which detailed descriptions of the star formation history and chemical enrichment are taken into account. In particular, we focus on the important role played stars of different chemical composition evolving along the red giant branch (RGB), horizontal branch (HB), asymptotic giant branch (AGB), and finally planetary nebula (PN) stages in relation to the sources of UV flux in elliptical galaxies. A few important applications of these new galaxy models are briefly outlined.
\end{abstract}

\section{Introduction}

In this paper, we will report on recent work on population synthesis in elliptical galaxies by Bressan et al. (1994, BCF), Tantalo et al. (1994, TCBF), and Fagotto (1994) that was particularly designed to explain the nature of the source of UV radiation detected in some of these systems and its dependence on basic quantities such as the $M g_{2}$ index and the velocity dispersion. In addition to this, we will report on preliminary results for model galaxies in suitable narrow band indices aimed at disentangling age from metallicity effects (Chiosi \& Bressan 1994).

\section{UV Excess in Elliptical Galaxies: the Sources}

Understanding the nature of the source of UV excess in elliptical galaxies has become possible thanks the recent developments in population synthesis models in which the detailed histories of star formation and metal enrichment are taken in to account. Following $B C F$, the UV excess and the detailed shape of the UV region of the spectra of elliptical galaxies with different level of UV emission are best interpreted by means of old, high metallicity stars in the so-called hot horizontal branch (H-HB) and AGB manqué phases (see also Greggio \& Renzini 1990). The ever occurring classical post AGB stars in their PN phase are found to play a minor role. Indeed, the observations by Ferguson et al. (1991) and Ferguson \& Davidsen (1993) show that the UV spectrum of UV loud galaxies requires a kind of source not hotter than about $25,000 \mathrm{~K}$, in contrast with the high temperature of the PN source (about 100,000 K).

It goes without saying that the detailed evolution during the core He-burning and later stages of low and intermediate mass stars of different chemical composition bears very much on their ability to become the source of UV radiation we are looking for.

The existence of H-HB and AGB manque stars is the complicate result of several concurring effects among which we recall:

a) Mass loss by stellar wind during the $R G B$ phase. For typical values of the age assigned to a galaxy (say $13 \mathrm{Gyr}$ ) and chemical abundances of the stellar population mix going from very low to solar, an unusually high rate of mass loss during the RGB phase can generate core He-burning stars of high effective temperature (cf. Dorman et al. 1994). This suggestion stems from the notion that the rate of mass loss increases with the metallicity much faster than currently assumed. The main drawback with this idea is that the kind of mass loss rate that is advocated has little observational support. The recent study of M67 by Liebert et al. (1994) is not conclusive in this respect.

b) Metal-poor, low mass stars of very old age. Low mass stars of low metallicity and normal mass loss during the RGB phase, getting very old (say at ages older than $20 \mathrm{Gyr}$ ) can undergo the core He-burning phase in the required range of effective temperatures (Lee 1994). The main difficulty with this possibility are the ages themselves, that are much older than commonly assigned to globular clusters $(13 \pm 2 \mathrm{Gyr})$. In addition to this, if the UV 
source resides in the HB stars of low metallicity, the question arises whether their luminosity and relative frequency are compatible with the observed spectral energy distribution (SPD) of elliptical galaxies in the range $2000<\lambda 3500 \AA$. Indeed the analysis of BCF has clarified that the SPDs seem to indicate that in the mix of stellar populations in an elliptical galaxy only very few stars with metallicity lower than about $\mathrm{Z}=0.008$ ought to exist (the analog of the G-Dwarf Problem in the solar vicinity). In contrast, as the luminosity of the HB stars of low metallicity is significantly fainter than the corresponding one of H-HB and AGB manqué stars (see Fagotto et al. 1994a,b), the relative number of such stars required to produce the total amount of UV flux could exceed the value suggested by the SED.

c) Enrichment Law $\Delta Y / \Delta Z$. Assuming the enrichment law $\Delta Y / \Delta Z=2.5$ (the solar case) and using normal rates of mass loss for the RGB phase, the appearance of the first generation of $\mathrm{H}-\mathrm{HB}$ and AGB manqué stars in single stellar population occurs at the age of about 7.6 Gyr. This occurs at even younger ages if $\Delta Y / \Delta Z$ is higher than 2.5 (see Fagotto 1994 and TCBF for details). This mechanism stands on the possibility that a certain fraction of the stellar content of an elliptical galaxy can reach metallicities as high as three to four times solar.

The models by BCF and TCBF show that the third alternative is the most plausible one. This is possible in galaxies of sufficiently high mass, which in virtue of the very efficient star formation rate possess higher values both for the mean and peak metallicity. According to the BCF and TCBF models, a few percent of the mass in stars stored in the highest metallicity bins can generate the desired amount of UV flux. In contrast galaxies of lower mass and lower mean and peak metallicity in turn, are not able to generate this type of stars and their UV flux is simply produced by their PN component. A natural result of this scheme is that the correlation of the UV flux with the $M g_{2}$ index and the velocity dispersion can be easily accounted for (see BCF, TCBF, and Chiosi \& Bressan 1994).

\section{The UV Excess as an Age Indicator}

BCF have suggested that the uprise in the UV flux as measured by the color $(1550-\mathrm{V})$ at the onset of the H-HB and AGB manqué stars can be used as probe of galaxy ages. In principle, different sources of UV radiation are dominant in the mix of stellar population at different ages. In the case of the sole PN stars, even if they can form early on in the galaxy life, their major contribution at the UV uprise is expected to occur at rather old ages, say at about 13-15 Gyr (BCF). In contrast, the H-HB and AGB manqué stars are formed much later but they soon dominate the UV flux. Their contribution is expected to occur at about $7.6 \mathrm{Gyr}$ for $\Delta Y / \Delta Z=2.5$ or equivalently a sudden change in the (1550V) color is expected to be observable at relatively low red-shifts, perhaps reachable with the present day space instrumentation. Detection of this change in the (1550-V) color or equivalently in the shape of the SED at a certain value of the red-shift would allow us to date a galaxy from the stellar clock running in the constituent stellar populations. The precise value of the red-shift corresponding to the above age depends on the particular model of the Universe in usage, namely the choice made for the Hubble constant $H_{0}$ and the deceleration parameter $q_{0}$ and finally the red-shift of galaxy formation $z_{f o r}$. Assuming the reasonable value of $z_{f o r}=5$ (see BCF for details and referencing), $H_{0}=50 \mathrm{~km} \mathrm{sec}-1$ $M \mathrm{pc}^{-1}$, the age of $7.6 \mathrm{Gyr}$ corresponds to the red-shift $z=0.81$ for $q_{0}=0$ and $z=0.34$ for $q_{0}=0.5$. The far reaching implications of dating galaxies by means of their $\mathrm{UV}$ properties are obvious (see also TCBF for further details on the subject).

\section{Is there a Real Age Range among Elliptical Galaxies ?}

The merge of the recent work on population synthesis in narrow band photometry $\left(H_{\beta}\right.$, $\mathrm{Mg}_{2},<\mathrm{MgFe}>$ indices) by Gonzales (1993) and Worthey (1994) and the models for elliptical galaxies in broad band photometry by BCF and TCBF allows us to tackle the question whether or not elliptical galaxies span a large range of ages. This problem has been addressed by Gonzales (1993) and Worthey (1994) by means of the evolutionary path of single stellar populations (SSP) in the so-called $H_{\beta}$ versus $\langle M g F e>$ plane. Galaxies 
fall in a rather narrow band comprised between evolutionary paths of SSPs with different metallicity (narrow range of $\langle M g F e\rangle$ ) and correspond to lines of different constant age. For the sake of illustration, M32 is at the young age side, while NGC4649 is at the old age side. Since the analysis was made using SSPs of given composition instead of galaxy models with ample range of metallicities, the above finding was subjected to the obvious criticism that in real galaxies it would not be possible to disentangle age from metallicity effects. Chiosi \& Bressan (1994) included the narrow band photometry in the models for elliptical galaxies of BCF and TCBF and found that as expected the galaxy paths run almost parallel to those of the SSPs and that all galaxies of the Gonzales (1993) sample are well matched by a unique sequence with mean metallicity slightly above solar and peak metallicity up to $\mathrm{Z}=0.08$ (the models with $M_{L}=3 \times 10^{12} M_{\odot}$ of BCF and TCBF). This means that all galaxies in the sample have nearly identical chemical structure. Since the model in question is expected to produce strong UV flux when older than 7.6 Gyr (indeed it was used by BCF and TCBF to match the SED of the UV loud galaxy NGC4649), if all galaxies were coeval and as old as say 13-15 Gyr, they would be sources of strong UV flux, in contrast with the observation. The easiest way to cope with this embarrassing result is to suppose that galaxies have different ages or more precisely that the bulk of star formation took place at different epochs. UV quiet galaxies like M32 do so because their stars are not old enough to become sources of strong UV flux via the H-HB and AGB manque channel. This conclusion is also consistent with the relation between the intensity of the UV flux and velocity dispersion. It seems that low velocity dispersion galaxies have been formed later than high velocity dispersion ones.

Acknowledgements This study has been financed by the Italian Ministry of University, Scientific Research and Technology (MURST) and the Italian Space Agency (ASI).

\section{References}

Bressan, A., Chiosi, C., \& Fagotto, F. 1994, ApJS 94, 63, BCF

Chiosi, C., \& Bressan, A. 1994, preprint

Dorman, B., O'Connell, R. W., Rood, R. T. 1994, ApJ, in press

Fagotto, F. 1994, In Stellar Populations, MSAIt in press

Fagotto, F., Bressan, A., Bertelli, G., \& Chiosi, C. 1994a, A\&AS, 104, 365

Fagotto, F., Bressan, A., Bertelli, G., \& Chiosi, C. 1994b, A\&AS, in press

Ferguson, H. C., \& Davidsen, A. F., 1993, ApJ; 408, 92

Ferguson, H. C., et al. 1991, ApJ, 382, L69

Gonzales, J. J. 1993, Ph.D. Thesis, Univ. California, Santa Cruz

Greggio, L., \& Renzini, A. 1990, ApJ 364, 35

Lee, Y.W. 1994, ApJ, 423, 248

Liebert, J. W., Saffer, R. A., Gree, E. M. 1994, A\&A, 225, L17

Tantalo, R., Chiosi, C., Bressan, A., \& Fagotto, F. 1994, preprint, TCBF

Worthey, G. 1994, ApJS, in press 